\title{
VARIABILIDAD ESPACIAL DE ATRIBUTOS FÍSICOS DE UN TYPIC HAPLUSTOX DE LOS LLANOS ORIENTALES DE COLÔMBIA
}

\section{LEONARDO RAMÍREZ-LÓPEZ ${ }^{1}$, AMPARO REINA-SÁNCHEZ ${ }^{1}$, JESÚS H. CAMACHO-TAMAYO}

\begin{abstract}
RESUMO: O objetivo deste trabalho foi estudar o comportamento da variabilidade espacial da resistência mecânica à penetração $(\mathrm{RP})$, da densidade do solo, da densidade de partículas do solo, da porosidade total e dos teores de areia, silte e argila, e as relações existentes entre as propriedades analisadas. O estudo foi desenvolvido em Puerto López (Meta-Colômbia). As amostras do solo foram coletadas em duas profundidades $(0-0,10$ e $0,10-0,20 \mathrm{~m})$, em intervalos regulares de $25 \mathrm{~m}$, em malha regular, compreendendo 42 pontos, sendo a RP medida até $0,42 \mathrm{~m}$ de profundidade. A RP não se correlacionou com nenhuma propriedade, na primeira profundidade. De 0,10 - 0,20 m de profundidade, a RP correlacionou-se com os teores de silte e areia. Os semivariogramas da RP não apresentaram correlação espacial nas duas profundidades, possivelmente devido ao sistema de preparo do solo, que modifica a dependência espacial na superfície, acarretando maior homogeneidade do solo. As propriedades ajustaram-se a modelos de semivariogramas exponenciais e esféricos. Para a profundidade de $0,10-0,20 \mathrm{~m}$, as propriedades não se ajustaram a modelos de semivariogramas, apresentando efeito pepita puro, ou seja, não apresentaram dependência espacial, à exceção da densidade aparente.
\end{abstract}

PALAVRAS-CHAVE: compactação, geoestatística, oxissol.

\section{SPATIAL VARIABILITY OF PHYSICAL ATTRIBUTES IN A TYPIC HAPLUSTOX IN THE EASTERN PLANES OF COLOMBIA}

\begin{abstract}
The objective of the present work was to determine the behavior of the spatial variability of the penetration resistance (RP), bulk density, soil density, total porosity and the sand, lime and clays contents, as well as the relation between these properties. The present study was conduced in Puerto López (Meta-Colombia). Soil samples were collected in a regular grid, comprising 42 points, spaced at separation distances of $25 \mathrm{~m}$ between points, for two depths $(0,00$ $0,10$ and $0,10-0,20 \mathrm{~m})$. The RP was analyzed up to the depth of $0,42 \mathrm{~m}$. The RP did not correlate with any property in the first depth. Between $0.10-0.20 \mathrm{~m}$ depth, the RP were correlated with the lime and sand contents. The RP semivariograms indicates no spatial correlation in both depths, possibly due to soil tillage practices, which modifies the superficial spatial structure, gradually inducing to soil homogeneity. The properties adjusted mainly to exponential and spherical semivariograms models. For $0,10-0,20 \mathrm{~m}$ depth, the properties presented nugget effect, without spatial correlation, except for the bulk density.
\end{abstract}

KEYWORDS: compaction, geostatistics, oxisol.

\section{INTRODUCCIÓN}

El diagnóstico y manejo de las propiedades físicas del suelo se hace desconociendo las características del terreno, sin considerar su condición y los requerimientos del cultivo, siendo importante realizar una evaluación previa dentro de los conceptos de sostenibilidad. Esta falta de conocimiento está conduciendo a seguir implementando sistemas de manejo de suelo que conducen

\footnotetext{
${ }^{1}$ Ingenieros Agrónomos, Facultad de Ciencias Agropecuarias, Universidad de Cundinamarca, Fusagasugá, Colombia, ia.leonardo@gmail.com.

${ }^{2}$ Ingeniero Agrícola, M.Sc., Profesor Asistente, Facultad de Ingeniería, Universidad Nacional de Colombia, Ciudad Universitaria, Edificio 214, Oficina 304, Bogotá, Colombia, jhcamachot@unal.edu.co

Recebido pelo Conselho Editorial em: 13-12-2006

Aprovado pelo Conselho Editorial em: 21-1-2008
} 
a la degradación (AMÉZQUITA, 2004). Básicamente los correctivos aplicados en situaciones involucradas con problemas físicos del suelo se resuelven mediante prácticas de mecanización, las cuales en algunas ocasiones se realizan de manera inadecuada, dejando de lado la inherencia de la variabilidad y dependencia espacial de las propiedades físicas de los suelos.

Esta variación ha conducido a revaluar el manejo tradicional que se realiza en el manejo de cultivos, donde la caracterización de la variabilidad espacial de las propiedades permite una mejor comprensión de las relaciones complejas entre las características del suelo y los factores ambientales (GOOVAERTS, 1998) y ayuda a determinar las prácticas de manejo apropiadas para el uso del suelo (PLANT, 2001). Además, la demanda de información representativa de la variabilidad espacial del suelo ha aumentado, desarrollándose modelos y sistemas de gerencia ambientales (GODWIN \& MILLER, 2003). BONGIOVANNI \& LOWENBERG-DEBOER, (2001), también hacen referencia al potencial de la agricultura de precisión para la protección del medio ambiente, no sólo para reducir tipos de contaminación derivada del uso inapropiado de diferentes insumos agrícolas, sino también para controlar la erosión y la compactación.

Atributos como la densidad aparente y la porosidad total muestran si un suelo presenta condiciones adecuadas para el desarrollo y exploración de las raíces, indicando si existen problemas de compactación. El método más común para determinar el grado de compactación de un suelo, es mediante el uso de un penetrómetro, el cual evalúa la resistencia mecánica que debe ejercer una raíz para explorar el suelo (BENGHOUGH \& MULLINS, 1990), siendo conveniente observar su relación con la humedad y la densidad aparente (VAZQUEZ et al., 1991), así como con la textura del suelo. Para un mejor entendimiento del comportamiento de estos atributos en el suelo, es conveniente verificar su variabilidad y dependencia espacial, mediante el uso de herramientas como la geoestadística, la estadística clásica y el kriging, con el fin de identificar zonas compactadas y realizar así un manejo localizado de este problema.

Dada la importancia del manejo de las propiedades física del suelo en la producción agrícola, así como la necesidad de entender el comportamiento, la relación de estas y la importancia del manejo de la compactación de los suelos, el objetivo del presente trabajo fue establecer la variabilidad espacial de la resistencia a la penetración (RP) en un oxisol de los llanos orientales, y su relación con la densidad aparente, la densidad real, la porosidad total y los contenidos de arena, limo y arcilla, para finalmente establecer zonas donde se pueden presentar limitantes para el desarrollo de cultivos.

\section{MATERIALES Y MÉTODOS}

El trabajo se realizó en Puerto López (Meta - Colombia), en la Estación Experimental Taluma (EET), de la Corporación Colombiana de Investigación Agropecuaria (CORPOICA), en un lote ubicado en las coordenadas geográficas $4^{\circ} 22^{\prime} 30^{\prime}$ ' de latitud norte y $72^{\circ} 13$ ' $52^{\prime \prime}$ de longitud oeste. La red de muestreo constó de 42 puntos (7 filas y 6 columnas), separados cada $25 \mathrm{~m}$, en un lote de 2,6 ha. El lote es considerado sabana nativa recién incorporada a la producción de soya, seis meses antes del muestreo.

El suelo es clasificado como un Typic Haplustox y en el momento del muestreo se encontraba con una humedad cercana a capacidad de campo y con una población vegetal en donde predominaban especies de la familia gramineae. En cada punto se tomaron muestras a dos profundidades, entre 0 y $0,10 \mathrm{~m}$ y entre 0,10 y $0,20 \mathrm{~m}$. Las variables medidas fueron resistencia a la penetración (RP), medido con penetrómetro digital Bush Recording Soil (Findley, Irvine, Midlothian, Scotland), con punta cónica cuya base es de 12,9 $\mathrm{mm}$ de diámetro; densidad aparente (Da), por el método del cilindro; densidad de partículas (Dr), por el método del picnómetro; porosidad total (PT) determinada a partir de la densidad de partículas y la densidad aparente; y contenidos de arena, limo y arcilla, por el método de Bouyucos. Para el caso de la RP, se analizó hasta una profundidad de $0,42 \mathrm{~m}$, cada $35 \mathrm{~mm}$, con el fin de identificar las profundidades en las cuales se registran valores de 0,9 y 1,5 $\mathrm{MPa}$, valores de referencia crítica para algunos cultivos, 
según lo indicado por CARTER (1988), realizando mapas para establecer la profundidad en que estas capas se encuentran, mediante interpolación del inverso cuadrado de las distancias.

Se analizaron los datos mediante estadística descriptiva para obtener la media, mediana, asimetría, curtosis, máximos y mínimos. También se determinó el coeficiente de variación (CV), el cual se puede tomar como un indicador de la magnitud de la variabilidad (ROQUE et al., 2005). Para la estadística descriptiva se empleó el programa SPSS 12.0. Para determinar los grados de dependencia espacial se tuvo en cuenta la teoría de variables regionalizadas, la cual dispone de diferentes métodos de análisis de variación espacial, siendo uno de ellos el semivariograma (VIEIRA, 2000; GIRALDO, 2002). Los semivariogramas de cada propiedad fueron obtenidos mediante el programa $\mathrm{GS}^{+}$(ROBERTSON, 1998), usando el procedimiento de autovalidación para la selección del modelo, técnica conocida como "Jack Knifing" (VIEIRA, 2000). Una vez determinada la dependencia espacial, se elaboraron los respectivos mapas de distribución utilizando el programa Surfer (Golden Software Inc., 1999) y se estableció la correlación lineal y espacial entre las propiedades analizadas.

\section{ANÁLISIS DE RESULTADOS}

Los valores similares de la media y la mediana para cada una de las propiedades estudiadas y los bajos valores de asimetría y curtosis indican que corresponden razonablemente a distribuciones normales (Tablas 1), situación confirmada por los valores de asimetría cercanos a cero, excepto para el contenido de arcilla en la primera profundidad. Comportamiento similar fue reportado para diferentes propiedades físicas del suelo por KILIÇ et al. (2004) y GREGO \& VIEIRA (2005). Esta aproximación a la distribución normal, expresado principalmente por la asimetría y la curtosis, aunque no es indispensable para el análisis geoestadístico, permiten una mayor confiabilidad, dado que la varianza es más estable y el semivariograma obtenido muestra una dependencia espacial mejor definida. De acuerdo con los criterios de WARRICK \&NIELSEN (1980) para el CV, las propiedades evaluadas mostraron baja variabilidad en las dos profundidades, con CV inferiores al $12 \%$, exceptuándose la RP a las dos profundidades, que presentó una mayor dispersión en la profundidad de 0-0,10 $\mathrm{m}$. Se observó también que la segunda profundidad presentó una menor dispersión respecto a la primera, que puede deberse a las labores de cultivo (manejo antrópico) así como a condiciones edafoclimáticas de la zona, donde el suelo está sometido a una tasa alta de intemperismo, que afecta principalmente la capa superficial (BUOL et al., 1997).

Los valores medios de la RP se encuentran por debajo de los reportados para la altillanura colombina por RUBIANO (2005) y JAIMES et al. (2003), quién en un estudio previo encontró valores de RP entre 1,73 y 3,37 MPa, para sabana nativa. Esto indica que la RP fue influenciada por el manejo de suelos, durante el cultivo de la soya y de la incorporación de enmiendas, prácticas que se realizan con arados disco y de cincel. Otro factor que influyó el comportamiento de la RP fue el contenido de humedad en el momento del muestreo, cercano a la capacidad de campo.

Los valores medios de la Dr son los comunes para este tipo de suelos. La Da denota valores medios, indicando la presencia de procesos naturales de adensamiento de los agregados del suelo (JAIMES et al., 2003). La PT presentó valores entre 40,02 y 41,83\%, que se ubican dentro del intervalo encontrado por JAIMES et al., (2003). Los contenidos de arena y limo son similares y superan el contenido de arcilla, que presentó valores entre 21,89 y $22,09 \%$, datos inferiores a $\operatorname{los}$ obtenidos por RUBIANO (2005) para este tipo de suelos.

Los contenidos de limo y arcilla no muestran correlación espacial. Por este motivo estas propiedades presentaron efecto pepita puro (EPP) para las dos profundidades analizadas, como se observa en la Tabla 2. Lo anterior indica que la dependencia espacial es baja o no significativa, por lo cual los mapas generados para estas propiedades no son representativos, al igual que todas que presentaron EPP, pero de igual manera se generaron mapas basados en un semivariograma lineal con pendiente uno, con el fin de identificar su correlación con las otras propiedades de suelo. 
TABLA 1. Medidas descriptivas de las variables RP (MPa), Dr $\left(\mathrm{g} \mathrm{cm}^{-3}\right), \mathrm{Da}\left(\mathrm{g} \mathrm{cm}^{-3}\right), \mathrm{PT}(\%)$, arena $(\%)$, limo (\%) y arcilla (\%), para dos profundidades del suelo. Descriptive statistics of soil properties RP (MPa), Dr $\left(\mathrm{g} \mathrm{cm}^{-3}\right)$, Da $\left(\mathrm{g} \mathrm{cm}^{-3}\right)$, PT $(\%)$, sand $(\%)$, silt $(\%)$ and clay $(\%)$, for two soil depth.

\begin{tabular}{lrrrrrrr}
\hline Parámetro & RP & Dr & \multicolumn{1}{c}{ Da } & PT & Arena & Limo & Arcilla \\
\cline { 2 - 7 } & \multicolumn{7}{c}{ Profundidad $0,00-0,10 \mathrm{~m}$} \\
Media & 0,30 & 2,44 & 1,43 & 41,36 & 37,37 & 40,54 & 22,09 \\
Mediana & 0,27 & 2,44 & 1,44 & 41,23 & 38,82 & 40,00 & 21,04 \\
Asimetría & 0,94 & $-0,26$ & $-0,52$ & 0,44 & $-0,54$ & 0,75 & 1,09 \\
Curtosis & 1,45 & $-0,59$ & 0,24 & $-0,09$ & $-0,83$ & $-0,07$ & 0,78 \\
Mínimo & 0,05 & 2,25 & 1,20 & 34,67 & 30,96 & 36,00 & 19,04 \\
Máximo & 0,76 & 2,59 & 1,59 & 51,14 & 42,12 & 48,00 & 29,88 \\
C.V. \% & 52,57 & 3,41 & 6,06 & 8,97 & 7,89 & 8,17 & 11,41 \\
\hline \multicolumn{7}{c}{ Profundidad $0,10-0,20 \mathrm{~m}$} \\
Media & 0,83 & 2,48 & 1,48 & 40,02 & 37,83 & 40,28 & 21,89 \\
Mediana & 0,81 & 2,47 & 1,49 & 39,64 & 38,96 & 40,00 & 21,04 \\
Asimetría & 0,43 & $-0,12$ & $-0,29$ & 0,11 & $-0,54$ & 0,40 & 0,75 \\
Curtosis & 0,02 & 0,11 & $-0,59$ & $-0,88$ & $-0,15$ & $-0,10$ & $-0,48$ \\
Mínimo & 0,51 & 2,31 & 1,38 & 34,88 & 30,12 & 34,00 & 19,04 \\
Máximo & 1,27 & 2,63 & 1,58 & 45,02 & 44,96 & 48,00 & 27,04 \\
C.V. \% & 20,98 & 2,83 & 3,54 & 6,87 & 8,53 & 8,00 & 10,38 \\
\hline
\end{tabular}

TABLA 2. Parámetros de los modelos de semivariogramas experimentales para las variables RP, Dr, Da, PT, arena, limo y arcilla, para dos profundidades del suelo. Parameters of the experimental semivariogram models for the soil properties RP, Dr, Da, PT, sand, silt and clay, for two soil depth.

\begin{tabular}{|c|c|c|c|c|c|}
\hline Parámetro & Modelo & \multicolumn{3}{|c|}{ Profundidad $0,00-0,10 \mathrm{~m}$} & $\mathrm{C}_{0} /\left(\mathrm{C}_{0}+\mathrm{C}\right)$ \\
\hline $\mathrm{RP}$ & Exponencial & 2,90 & 8,81 & 375,80 & 0,33 \\
\hline Dr & Exponencial & 0,0004 & 0,0070 & 11,10 & 0,06 \\
\hline $\mathrm{Da}$ & Exponencial & 0,0049 & 0,0158 & 349,30 & 0,31 \\
\hline PT & Exponencial & 0,86 & 13,03 & 8,60 & 0,07 \\
\hline Arena & Esférico & 0,70 & 8,97 & 9,70 & 0,08 \\
\hline Limo & EPP & 6,47 & & & \\
\hline Arcilla & EPP & 12,16 & & & \\
\hline \multicolumn{6}{|c|}{ Profundidad $0,10-0,20 \mathrm{~m}$} \\
\hline $\mathrm{RP}$ & EPP & 5,18 & & & \\
\hline Dr & EPP & 0,0051 & & & \\
\hline $\mathrm{Da}$ & Esférico & 0 & 0,00267 & 37,90 & 0,00 \\
\hline PT & EPP & 7,58 & & & \\
\hline Arena & EPP & 10,82 & & & \\
\hline Limo & EPP & 5,10 & & & \\
\hline Arcilla & EPP & 6,40 & & & \\
\hline
\end{tabular}

La Da fue la única propiedad que se ajustó a modelos en las dos profundidades (Figura 1), con un modelo exponencial y dependencia moderada para la primera profundidad. Para la profundidad entre 0,10 y $0,20 \mathrm{~m}$ se ajustó a un modelo esférico, mostrando una fuerte dependencia espacial (CERRI et al., 2004). En esta misma profundidad la Dr, PT y contenido de arena presentaron rangos bajos y una dependencia espacial fuerte, pero se observa que en la segunda profundidad esta dependencia espacial desaparece. Los rangos y la estructura espacial encontrados, muestran un comportamiento similar en suelos con bajos contenidos de arcillas, en estudios realizados por KILIÇ et al. (2004), CARROLL \& OLIVER (2005). 
También se observa que el rango obtenido para la Da y la RP son superiores a la máxima distancia de muestreo $(195,3 \mathrm{~m})$, indicando que estos parámetros muestran dependencia espacial a mayores distancias, siendo necesario la realización de muestreos en áreas mayores, con distancias que sean menores a la máxima distancia utilizada en el presente estudio, dada la condición de la teoría de variables regionalizadas (VIEIRA, 2000), cuyos resultados son solo válidos para puntos internos de la zonas donde se hace el muestreo.

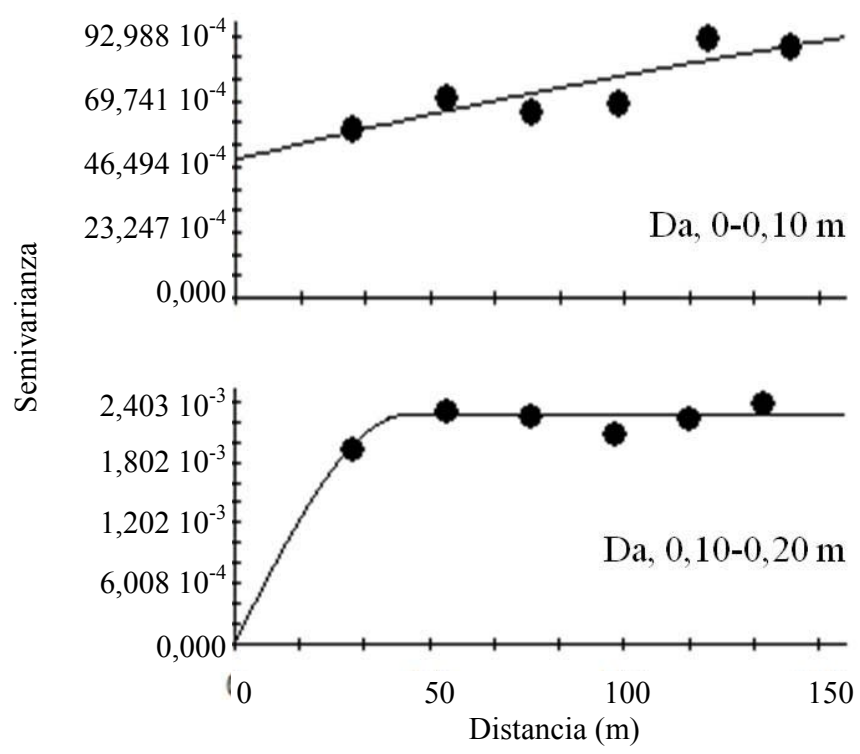

FIGURA 1. Semivariogramas de la densidad aparente (Da), a las profundidades de $0-0,10 \mathrm{~m}$ y 0,10 - 0,20 m. Semivariograms for soil bulk density (Da), for 0-0,10 $\mathbf{m}$ and $\mathbf{0 , 1 0 - 0 , 2 0 ~} \mathbf{m}$ depth.

La RP en los primeros $0,10 \mathrm{~m}$ de profundidad se ajustó a un modelo exponencial, con dependencia espacial moderada y de acuerdo con AMÉZQUITA (1998), quien referencia como crítica la máxima presión radical ejercida por la mayoría de cultivos, entre 0,9-1,5 MPa, en ningún sitio se encontraron valores restrictivos a esta profundidad (Figura 2). También se observó correlación espacial entre las dos profundidades, donde zonas con mayor RP en la primera profundidad, presentan algún grado de correspondencia con zonas de mayor RP en la segunda, mostrando una continuidad en las condiciones del suelo a medida que se profundiza.

En la profundidad de 0,10-0,20 $\mathrm{m}$ se presentan restricciones para algunos cultivos, dado que ella supera valores de $0,9 \mathrm{MPa}$, con un área cercana al 50,5\%, como se observa en la Figura 3. Para la capa comprendida entre 0,20 y 0,30 m los valores de RP de 0,9 MPa se presentan en el 48,3\% del terreno. Para la RP de 1,5 MPa (Figura 3) se encontró que el 44,4\% del área se situó entre 0,20 y $0,30 \mathrm{~m}$ de profundidad, un $44,6 \%$ del área se situó entre 0,30 y $0,40 \mathrm{~m}$ de profundidad y en el $11 \%$ del terreno la capa se situó por debajo de los 0,40 $\mathrm{m}$ de profundidad. Es de esperar que en los sitios en los que las capas compactadas se encuentran a una mayor profundidad, el sistema radical de los cultivos exploren un mayor volumen de suelo, resultando en un mejor anclaje de las raíces y desarrollo del cultivo.

La Da presenta valores comunes para este tipo de suelos, sin embargo, se presentó una zona donde los valores de densidad aparente en los primeros $0,10 \mathrm{~m}$ de profundidad del suelo están por encima de $1,45 \mathrm{~g} \mathrm{~cm}^{-3}$, que representa el 40,23\% del área, concluyendo que esta zona presenta problemas de compactación, donde también se presentó una PT baja.

La Da y la PT (Tabla 3) presentan correlación lineal altamente significativa, que de igual manera se puede corroborar en la Figura 4, donde al contrastar los mapas de estas propiedades se observa que zonas con alta Da corresponden a zonas de baja PT, para la dos profundidades. Comportamiento similar se presenta entre la Dr y la PT. 

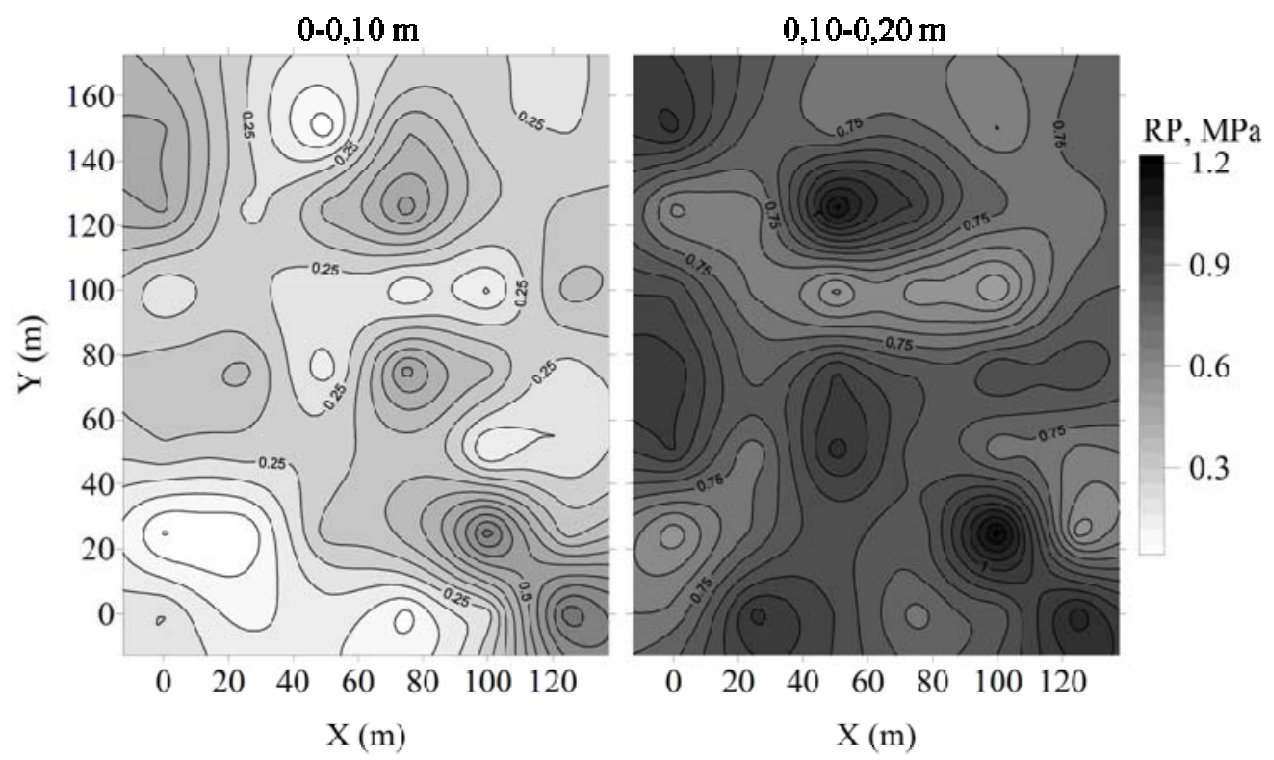

FIGURA 2. Mapas de resistencia a la penetración para la EET. Resistance penetration maps for EET.
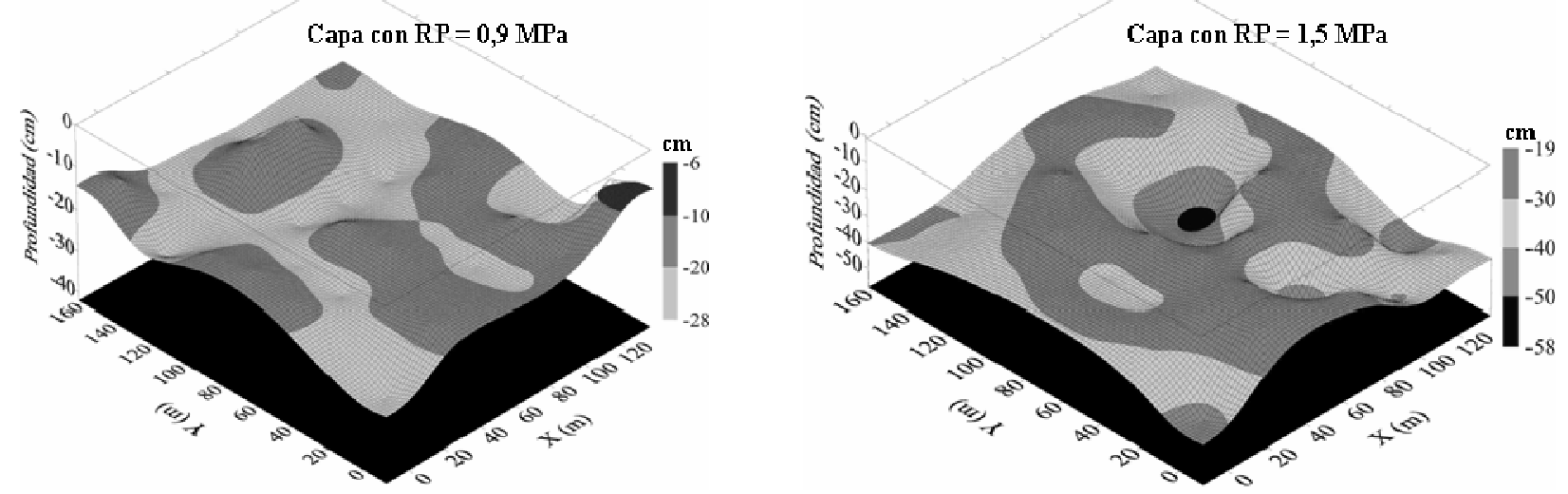

FIGURA 3. Profundidades de la capas del suelo con resistencia a la penetración de 0,9 y 1,5 MPa. Soil level surface depths with penetration resistance of 0,9 and 1,5 MPa.

TABLA 3. Correlación de Pearson de las variables RP, Dr, Da, PT, arena, limo y arcilla, para dos profundidades del suelo. Pearson correlation of the soil properties RP, Dr, Da, PT, sand, silt and clay, for two soil depth.

\begin{tabular}{|c|c|c|c|c|c|c|c|}
\hline Parámetro & $\mathrm{RP}$ & Dr & $\mathrm{Da}$ & PT & Arena & Limo & Arcilla \\
\hline \multicolumn{8}{|c|}{ Profundidad $0,00-0,10 \mathrm{~m}$} \\
\hline $\mathrm{RP}$ & 1 & & & & & & \\
\hline Dr & $-0,001$ & 1 & & & & & \\
\hline $\mathrm{Da}$ & $-0,031$ & 0,198 & 1 & & & & \\
\hline PT & 0,037 & $0,360 *$ & $-0,842 * *$ & 1 & & & \\
\hline Arena & 0,051 & $-0,224$ & $-0,022$ & $-0,099$ & 1 & & \\
\hline Limo & $-0,154$ & $0,366^{*}$ & 0,071 & 0,129 & $-0,682 * *$ & 1 & \\
\hline Arcilla & 0,143 & $-0,219$ & $-0,067$ & $-0,054$ & $-0,273$ & $-0,517 * *$ & 1 \\
\hline \multicolumn{8}{|c|}{ Profundidad $0,10-0,20 \mathrm{~m}$} \\
\hline $\mathrm{RP}$ & 1 & & & & & & \\
\hline Dr & $-0,254$ & 1 & & & & & \\
\hline $\mathrm{Da}$ & $-0,154$ & $-0,02$ & 1 & & & & \\
\hline PT & $-0,041$ & $0,637 * *$ & $-0,783 * *$ & 1 & & & \\
\hline Arena & $0,353^{*}$ & $-0,387^{*}$ & $-0,121$ & $-0,143$ & 1 & & \\
\hline Limo & $-0,338^{*}$ & $0,548 * *$ & 0,205 & 0,184 & $-0,751 * *$ & 1 & \\
\hline Arcilla & $-0,023$ & $-0,226$ & $-0,118$ & $-0,058$ & $-0,355^{*}$ & $-0,350 *$ & 1 \\
\hline
\end{tabular}

*La correlación es significativa a un nivel de 0,$05 ; * *$ La correlación es significativa a un nivel de 0,001 

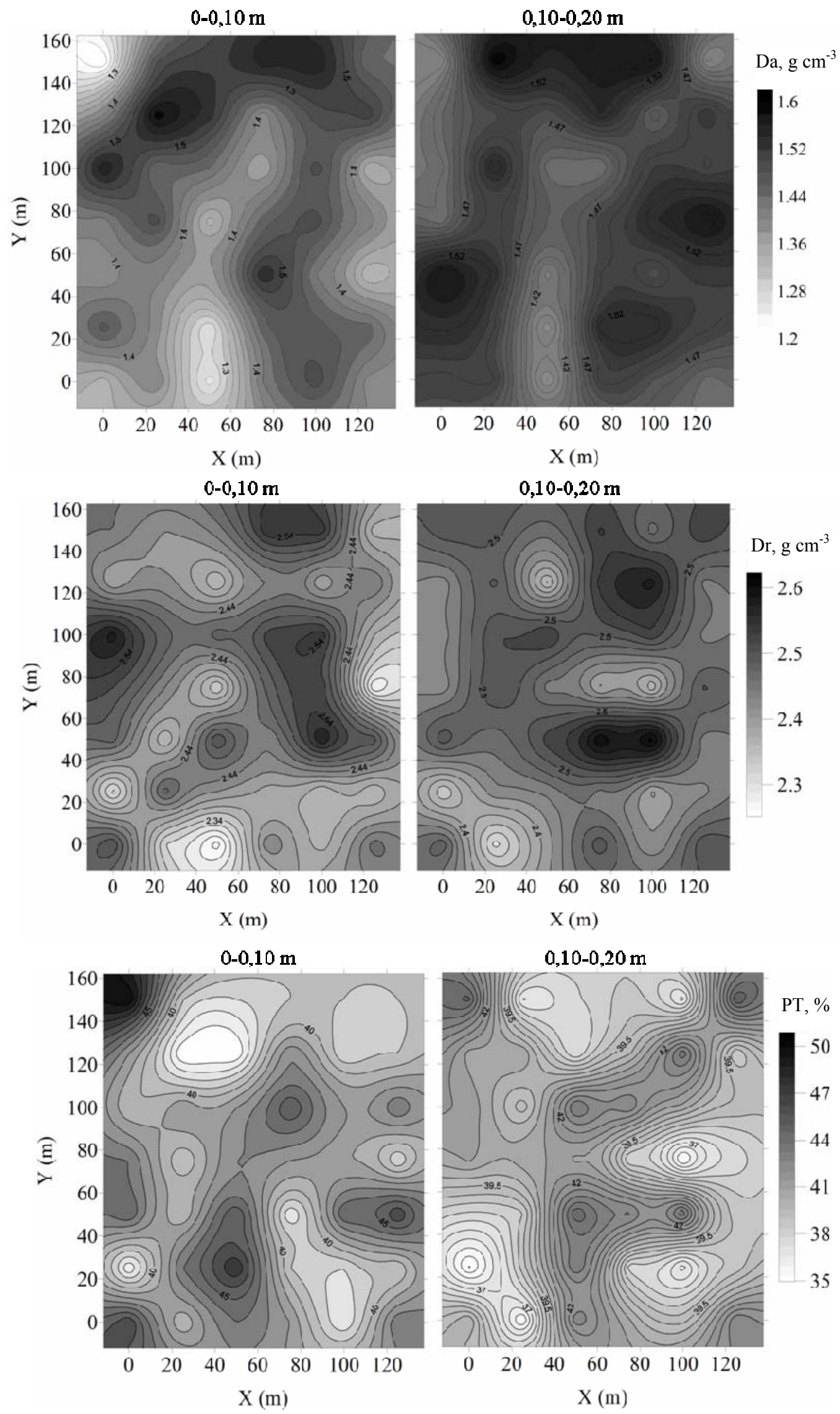

FIGURA 4. Mapas de distribución de la densidad aparente (Da), densidad de partículas (Dr) y porosidad total (PT), para la Estación Experimental Taluma. Distribution maps of bulk density (Da), particle density (Dr) and total porosity (PT), for Taluma Experimental Station. 
La Dr correlacionó positivamente con el contenido de limo en las dos profundidades, indicando que su valor depende en gran medida de ésta partícula del suelo, pero también muestra una correlación negativa con el contenido de arena para la profundidad entre 0,10 y $0,20 \mathrm{~m}$. La arcilla solo presentó correlación con los contenidos de limo y no presentó correlación significativa con las otras propiedades del suelo. Este comportamiento de la arcilla se debe en gran parte a que los contenidos de arena y limo son mayores.

Aunque la RP no presentó correlación significativa con ninguna propiedad en la primera profundidad, si muestra una correlación significativa con los contenidos de arena y limo para la profundidad entre 0,10 y 0,20 m. Por otra parte, al comparar los mapas de RP (Figura 2) con los mapas de Da y PT (Figura 4), se observa que zonas que presentan altos valores de RP, corresponden a zonas de baja Da y alta PT.

\section{CONCLUSIONES}

Las propiedades físicas del suelo se aproximan a una distribución normal, mostrando baja dispersión para las dos profundidades, exceptuándose la resistencia a la penetración, que presentó alta variabilidad.

La resistencia a la penetración, la densidad real, la porosidad total y los contenidos de arena solo presentaron dependencia espacial a una profundidad entre 0 y $0,10 \mathrm{~m}$. De igual manera, a la profundidad entre $0,10-0,20 \mathrm{~m}$ no presentaron dependencia espacial, mostrando una variabilidad espacial aleatoria.

Los mapas que se obtienen a partir del kriging evidencian correlación espacial entre las diferentes propiedades del suelo, sin embargo cuando los resultados obtenidos presentan modelos de semivariogramas con efecto pepita puro y/o con dependencia espacial baja, la confiabilidad de los datos estimados disminuye.

El uso de la geoestadística permite identificar capas compactadas en el suelo, así como la localización de zonas adensadas que pueden restringir el desarrollo radical de diferentes cultivos, siendo por tanto una herramienta adecuada para tomar decisiones y facilitar un manejo localizado de capas compactadas.

\section{REFERENCIAS}

AMÉZQUITA, E. Hacia la sostenibilidad de los suelos en los Llanos Orientales de Colombia. In: CONGRESO COLOMBIANO DE LA CIENCIA DEL SUELO, 9., 1998, Paipa. Memorias... Paipa, Colombia: Sociedad Colombiana de la Ciencia del Suelo, 1998. p.106-20.

AMÉZQUITA, E. La fertilidad física del suelo. In: CONGRESO LATINOAMERICANO Y DE LA CIENCIA DEL SUELO, 16., 2004, Cartagena. Memorias... Cartagena, Colombia: Sociedad Colombiana de la Ciencia del Suelo, 2004. 1 CD-ROM.

BENGHOUGH, A.G.; MULLINS, C.E. Mechanical impedance to root growth: a review of experimental techniques and root growth responses. Journal of Soil Science, Oxford, v.41, p.34158, 1990.

BONGIOVANNI, R.; LOWENBERG-DEBOER, J. Precision Agriculture: economics of nitrogen management in corn using site-specific crop response estimates from a spatial regression model. In: AMERICAN AGRICULTURAL ECONOMISTS ASSOCIATION ANNUAL MEETING, 2001, Chicago, Illinois.

BUOL, S.W.; HOLE, F.D.; McCRACKEN, R.J.; SOUTHARD, R.J. Soil genesis and classification. Iowa: Iowa State University Press, 1997. 527 p.

BURROUGH, P.A. Soil variability: a late 20th century view. Soils and Fertilizers, New York, v.56, n.5, p.529-62, 1993. 
CARROLL, Z.L.; OLIVER, M.A. Exploring the spatial relations between soil physical properties and apparent electrical conductivity. Geoderma, Amsterdan, v.128, n.3-4, p.354-74, 2005.

CARTER, M. Penetration resistance to characterize the depth and persistence of soil loosening tillage studies. Canadian Journal of Soil Science, Ottawa, v.68, n.4, p.657-68, 1988.

CERRI, C.E.P.; BERNOUX, M.; CHAPLOT, V.; VOLKOFF, B.; VICTORIA, R.L.; MELILLO, J.M.; PAUSTIAN, K.; CERRRI, C.C. Assessment of soil property spatial variation in an Amazon pasture: basis for selecting an agronomic experimental area. Geoderma, Amsterdan, v.123, n.1-2, p.51-68, 2004.

GIRALDO, H, R. Introducción a la geoestadística. Universidad Nacional de Colombia. Medellín: Facultad de Ciencias, Universidad Nacional de Colombia, 2002. Mimeografiado.

GODWIN, R.J.; MILLER, P.C.H. A review of the technologies for mapping within-field variability. Biosystems Engeneering, United Kingdon, v.84, n.4, p.393-407, 2003.

GOOVAERTS, P. Geostatistical tools for characterizing the spatial variability of microbiological and physico-chemical soil properties. Biology and Fertility of Soils, Berlin, v.27, n.4, p.315-34, 1998.

GREGO, C.R.; VIEIRA, S.R. Variabilidade espacial de propriedades físicas do solo em uma parcela experimental. Revista Brasileira de Ciência do Solo, Viçosa, v.29, n.2, p.169-77, 2005.

JAIMES, W.; NAVAS, G.; SALAMANCA, C.; CONDE, A. Estudio Detallado de Suelos de la Estación Experimental de CORPOICA "Sabanas" en la Altillanura Colombiana. 2003.

KILIÇ, K.; ÖZGÖZ, E.; AKBAŞ, F. Assessment of spatial variability in penetration resistance as related to some soil physical properties of two fluvents in Turkey. Soil and Tillage Research, Amsterdam, v.76, n.1, p.1-11, 2004.

PLANT, R.E. Site-specific management: the application of information technology to crop production. Computers and Electronics in Agriculture, Oxford, v.30, n.1-3, p.9-29, 2001.

ROBERTSON, G.P. $G S^{+}$: Geostatistis for the environmental sciences - GS ${ }^{+}$user's guide. Plainwell: Gamma Desing Software, 1998. 152 p.

ROQUE, M.W.; SILVA, E.L.; MATSURA, E.E. Análisis comparativo de la dependencia espacial de propiedades físicas e hídricas en suelos de un transecto perpendicular a un canal de drenaje. In: Samper, J.; Paz, A. Estudios de la zona no saturada del suelo. La Coruña: Universidad de La Coruña, 2005. v.7, p.229-33.

RUBIANO, Y. Sistema georeferenciado de indicadores de calidad del suelo. Herramienta SIG para apoyo a la planificación, uso y manejo del suelo. 2005. $128 \mathrm{f}$. Tesis (Doctorado em Ciencias Agropecuárias - Manejo de Suelos y Aguas) - Universidad Nacional de Colombia, Palmira, 2005.

VAZQUEZ, L.; MYHRE, D.L.; HANLON, E.A.; GALLAHER, R.N. Soil penetrometer resistance and bulk density relationships after long-term no tillage. Communications in Soil Science and Plant Analysis, New York, v.22, n.19-20, p.2101-17, 1991.

VIEIRA, S.R. Geoestatística em estudos de variabilidade espacial do solo. In: NOVAIS, R.F. de; ALVAREZ V.H.; SCHAEFER, C.E.G.R. Tópicos em ciência do solo. Viçosa - MG: Sociedade Brasileira de Ciência do Solo, 2000. v.1, p.1-54.

WARRICK, A.W.; NIELSEN, D.R. Spatial variability of soil physical properties in the field. In: HILLEL, D. Applications of soil physics. New York: Academic Press, 1980. p.319-44. 